Journal of Mathematics and Informatics

Vol. 9, 2017, 19-27

ISSN: 2349-0632 (P), 2349-0640 (online)

Published 17 October 2017

www.researchmathsci.org

DOI: http://dx.doi.org/10.22457/jmi.v9a3

Journal of

Mathematics and

Informatics

\title{
Fixed-Formation Control Based on Event-Driven of Multi-Agent System
}

\author{
Zheng Yu-lai \\ College of Mathematics and Physics \\ Chongqing University of Posts and Telecommunications \\ Chongqing, China. E-mail: 441287932@qq.com
}

Received 30 September 2017; accepted 17 October 2017

\begin{abstract}
In this paper, we study the formation control problem of the second-order multi-agent system, the so-called formation control problem is solved by designing decentralized control rate. All the agents are formed by autonomous movement to achieve a given formation. Considering that the multi-agent consumes in communication. In this paper, an event-driven control law protocol is proposed to reduce the number of multi-agent sampling times in the same time, and the sufficient conditions for the given formation to be satisfied in the range of the multi-agent system are given. Finally, the correctness and validity of the event-driven control protocol are verified by numerical simulation experiments.
\end{abstract}

Keywords: Multi-agent systems; time-varying formation control; event-based

\section{AMS Mathematics Subject Classification (2010): 03C10, 05CXX}

\section{Introduction}

With the extensive application of multi-agent in science and engineering, the research of multi-agent algorithm has aroused extensive research interests of scholars. The problem on formation control problems is how to make multi-agent regulate their own behavior, so as to form the desired shape or reach the desired target point. Formation control is an important research direction of multi-agent coordination control system. It is used extensively in nature, such as ant formed colony formation to carry food, wolves formed a certain formation to hunt prey, migratory birds, and so on. In real life, there are many applications, such as UAV formation [1], environmental monitoring and transportation [2,3], rescue and investigation [4]. Multi-agent formation control can replace human beings to accomplish complex and dangerous tasks. Because of its extensive practical application, multi-agent and multi-robot system formation has obtained a lot of research results. At present, the formation control method can be divided into the following two categories: leader follower method [5,6], structure based on virtual behavior method[7,8]. Wang et al. [9] proposed a UAV hierarchical control strategy to form a constant formation in obstacle avoidance conditions. Gu et al. [10] adopt the leader-follower method researching UAV frame system is studied UAV system and the two fixed wing UAV flight experiments are carried out to verify the validity of the method. Bayezit and Fidan [11] proposed the formation control of UAVs based on virtual structure in three-dimensional 


\section{Yu-lai Zheng}

space, and they validated the effectiveness of the control protocol by numerical experiments. Although the leader follower, the virtual structure and the behavior based approach can solve the formation control problem of the UAV framework system. Beard et al. [12] point out that these methods are also flawed. For example, the leader follower method achieves the given position according to the distributed control but lack of robustness and the availability of neighborhood information of the leader. Behavior based formation method is not suitable for distributed implementation because it requires a great deal of communication and computation. Wang et al. [13] study the formation control of a two order multi-agent system with a leader under directed graphs, and they give the sufficient conditions for the formation of the invariant formation of the multiagent. Chen et al. [14] study the UAV system under switching topology. A Lyapunov function is constructed according to the algebraic Riccati equation. And they give the necessary and sufficient conditions for the formation of time varying formation and prove the validity of the theory through experiments.

The research on the formation problem is considered under the continuous time. However, in practical applications, as individuals are limited by resources, energy, and so on, it is necessary to reduce the individual energy consumption as much as possible. With the development of computer communication technology and the rapid development of digital sensor, microprocessor and other industrial technology, to design distributed control algorithm and to achieve the desired effect for each agent become more practical. The agent has quiet difficult in obtaining global information. Hence, there is a need to communicate with each other to require more intelligence to save energy consumption. Therefore, event-driven control can achieve the above requirements. When a control exceeds a given threshold, the system updates the controller. This control method reduces the number of updates of the controller, saves the resource and reduces the energy consumption of the intelligent controller by reducing the number of updates. At present, the event-driven control mechanism has attracted the attention of a large number of scholars and has gained a lot of research results. Seyboth et al. use independently the event-driven function of the state to determine the corresponding event driven time series, and study the average consistency of first and two order multi-agent systems. Zhu et al. [15] research consistency of the general linear model of event-driven control with input delay based on the design of the new control protocol, and they define a new error function and driving function and ensure that the system does not exist the Zeno-behavior. Guzey [16] drives itself to a pre-set desired state via a distributed controller by communicating location and speed information at the event based sampling time with the neighbors.

This paper studies the multi-agent system under the event-driven control. The paper is organized as follows. In section 2, we give brief review of mathematical preliminaries. In section 3, we study the multi-agent system under the event-driven control. Section 4 gives sufficient conditions of reaching a given position within a certain range of error using the relative position and velocity between adjacent agent and theoretically excludes Zeno-behavior. In section 5, the correctness and validity of the event-driven control protocol are verified by numerical simulation experiments. Section 6 contains conclusions. 


\section{A Fixed-Formation Control Based on Event-Driven of Multi-Agent System}

\section{Mathematical preliminaries}

Suppose that $G=(V, F, A)$ is a graph containing $\mathrm{N}$ nodes. Where $v=\left\{v_{1}, v_{2} \cdots v_{n}\right\}$ is set of nodes. For the graph, $v_{i}$ represents the $i$ agent. Define the neighbor set of $i$ as $N_{i}=\{j \in v:(j, i) \in \xi\}$, That is to say, $\mathrm{v}_{i}$ can receive the information of $v_{j}$ and $a_{i j}>0 \cdot\left|N_{i}\right|$ represents the number of adjacent points of $\mathrm{v}_{i}$. Suppose there are no rings or parallel edges in the graph. namely, when $i=j, a_{i j}=0 . F \subset v \times v$ represents an edge set of multi-agent. $A=\left[a_{i j}\right]_{N \times N}$ is an adjacency matrix. $D=\operatorname{diag}\left\{d_{1}, d_{2} \cdots d_{n}\right\}$ is degree matrix whose diagonal elements are defined by $d_{i}=\sum_{j \in N_{i}} a_{i j}, L$ The Laplacian of the weighted digraph $\mathrm{G}$ is defined as $L=D-A$. It is well-known that $L$ has exactly one zero eigenvalue and all the other eigenvalues have positive real parts if and only if the digraph $\mathrm{G}$ has a directed spanning tree. In this paper, without loss of generality, we assume that node 1 is the root of the directed spanning tree.

\section{Model description}

In this paper, two order multi-agent systems are considered, and the dynamics equations of each agent are expressed as follows

$$
\left\{\begin{array}{l}
\dot{x}_{i}=v_{i} \\
\dot{v}_{i}=u_{i}
\end{array}\right.
$$

where $x_{i}(t), v_{i}(t), u_{i}(t)$ respectively denote locations, the speed, the control input of multiple agents.

In this article, our control objection is to allow each agent to reach a given position and speed in a given range of errors. Namely, $x_{i}-p_{i d} \rightarrow \Delta 、 v_{i}-p_{i v} \rightarrow \Delta$. In order to save resources, the event- based sampling control is introduced. Assume that the agent $i$ only sample in the driving moment $t_{0}=t_{0}^{i}, t_{1}^{i} \cdots, t_{k}^{i} \cdots$ by the neighbors and state information of each agent in the state. Therefore, the following distributed sampling control is designed for each multi-agent

$$
\begin{aligned}
u_{i}(t)= & -\sum_{j \in N_{i}} a_{i j}\left[\alpha\left(x_{i}\left(t_{k}^{i}\right)-p_{i d}-\left(x_{j}\left(t_{k^{j}}^{j}\right)-p_{j d}\right)\right)\right. \\
& \left.+\beta\left(v_{i}\left(t_{k}^{i}\right)-v_{j}\left(t_{k^{j}}^{j}\right)\right)\right]
\end{aligned}
$$

where $p_{i d}$ is the target position of agent $i$, for each agent $i$, we define the driving time series as $t_{k+1}^{i}=\inf \left\{t>t_{k}^{i}: f_{i}\left(t, e_{i x}(t), e_{i v}(t)\right)>0\right\}$, where the driving function is

$$
\begin{aligned}
f_{i}\left(t, e_{i x}(t), e_{i v}(t)\right) & =\sqrt{\alpha(\alpha+\beta)}\left|e_{i x}\right|+\sqrt{\beta(\alpha+\beta)}\left|e_{i v}\right| \\
& -\left|p_{i v}\right|-e^{-\rho\left(t-t_{0}\right)}
\end{aligned}
$$

where 
Yu-lai Zheng

$$
e_{i x}(t)=x_{i}\left(t_{k}^{i}\right)-x_{i}(t), e_{i v}(t)=v_{i}\left(t_{k}^{i}\right)-v_{i}(t), \forall t \in\left[t_{k}^{i}, t_{k+1}^{i}\right) .
$$

The control strategy (2) can be rewritten as

$$
\begin{aligned}
u_{i}(t)= & -\sum_{j \in N_{i}} a_{i j}\left[\alpha \left(e_{i x}(t)+x_{i}(t)-p_{i d}-\left(e_{j x}(t)+x_{j}(t)-p_{j d}\right)\right.\right. \\
& \left.+\beta\left(e_{i v}(t)+v_{i}(t)-\left(e_{j v}(t)+v_{j}(t)\right)\right)\right]
\end{aligned}
$$

suppose

$$
\begin{aligned}
& \varepsilon_{i}(t)=x_{i}(t)-p_{i d} \\
& \eta_{i}(t)=v_{i}(t)-p_{i v}
\end{aligned}
$$

For (3.5) derivation, we substitute it into (3.4). we obtain

$$
\begin{aligned}
\dot{\varepsilon}_{i}(t)= & \eta_{i}(t)+p_{i v} \\
\dot{\eta}_{i}(t)= & -\sum_{j=1}^{N} a_{i j}\left[\alpha\left(e_{i x}(t)-e_{j x}(t)+\varepsilon_{i}(t)-\varepsilon_{j}(t)\right)\right. \\
& \left.+\beta\left(e_{i v}(t)-e_{j v}(t)+\eta_{i}(t)-\eta_{j}(t)\right)\right]
\end{aligned}
$$

Then, using the Kronecker product representation, (3.6) can be rewritten in the compact form

$$
\begin{aligned}
& \dot{\varepsilon}(t)=\eta(t)+p_{v} \\
& \dot{\eta}(t)=-\alpha L\left(\varepsilon(t)+e_{x}(t)\right)-\beta L\left(\eta(t)+e_{v}(t)\right)
\end{aligned}
$$

where

$\mathcal{E}(t)=\left[\varepsilon_{1}(t), \varepsilon_{2}(t) \cdots \varepsilon_{N}(t)\right]^{\prime}, \eta(t)=\left[\eta_{1}(t), \eta_{2}(t) \cdots \eta_{N}(t)\right]^{\prime}, P_{v}=\left[\begin{array}{llll}p_{1 v}, p_{2 v} & \cdots & p_{N v}\end{array}\right]^{\prime}$. let $z(t)=\left(\varepsilon^{T}(t), \eta^{T}(t)\right)^{T}$, we can get

$$
\dot{z}(t)=\left[\begin{array}{cc}
0 & I_{N} \\
-\alpha L & -\beta L
\end{array}\right] z(t)+\left[\begin{array}{c}
p_{v} \\
-\alpha L e_{x}(t)-\beta L e_{v}(t)
\end{array}\right]
$$

Furthermore, we can get

$$
z(t)=e^{M\left(t-t_{0}\right)} z\left(t_{0}\right)+\int_{t_{0}}^{t} e^{M(t-\tau)} Q d \tau
$$

where

$$
M=\left[\begin{array}{cc}
0 & I_{N} \\
-\alpha L & -\beta L
\end{array}\right], Q=\left[\begin{array}{c}
p_{v} \\
-\alpha L e_{x}(t)-\beta L e_{v}(t)
\end{array}\right]
$$

\section{Consensus in a given range of errors}

Lemma 4.1. if $\alpha, \beta>0$, then exist $k_{1}, k_{2}>0,\left\|e^{M\left(t-t_{0}\right)}\right\| \leq k_{1} e^{-k_{2}\left(t-t_{0}\right)}$ is available.

Lemma 4.2. For any vector, $x^{T} y \leq \frac{x^{T} x+y^{T} y}{2}$ is founded.

Theorem 4.1. Consider the multi-agent systems (3.1) with the control strategy (3.2) and the triggering time instants determined by (3.3).for any $\alpha, \beta>0$, $\mathrm{k}_{2}>\rho$, Multiple intelligences form target formations within the range of error $\Delta$ where

$$
\Delta=\frac{(m+1) k_{1}}{k_{2}}\left\|p_{v}\right\| P_{v}=\left[p_{1 v}, p_{2 v} \cdots p_{N_{v}}\right]
$$


A Fixed-Formation Control Based on Event-Driven of Multi-Agent System

Proof: $\|z(t)\| \leq k_{1} e^{-k_{2}\left(t-t_{0}\right)}\left\|z\left(t_{0}\right)\right\|+k_{1} \int_{t_{0}}^{t} e^{-k_{2}\left(t-t_{0}\right)}\|Q\| d \tau$

According to the definition of $Q$, we can get

$$
\begin{aligned}
Q^{T} Q & =p_{v}^{T} p_{v}+\alpha^{2} e_{x}^{T} L^{T} L e_{x}+\alpha \beta e_{x}^{T} L^{T} L e_{v} \\
& +\alpha \beta e_{v}^{T} L^{T} L e_{x}+\beta^{2} e_{v}^{T} L^{T} L e_{v} .
\end{aligned}
$$

Set $m$ is spectral radius of matrix $L^{T} L$.

$$
\begin{aligned}
\|Q\| \leq & \left(p_{v}^{T} p_{v}+\alpha^{2} e_{x}^{T} L^{T} L e_{x}+\alpha \beta \frac{e_{x}^{T} L^{T} L e_{x}+e_{v}^{T} L^{T} L e_{v}}{2}\right. \\
& \left.+\alpha \beta \frac{e_{x}^{T} L^{T} L e_{x}+e_{v}^{T} L^{T} L e_{v}}{2}+\beta^{2} e_{v}^{T} L^{T} L e_{v}\right)^{1 / 2}
\end{aligned}
$$

According to lemma 1, we can obtain

Therefore, we get

$$
\|Q\| \leq \sqrt{m \alpha(\alpha+\beta)}\left\|e_{x}\right\|+\sqrt{m \beta(\alpha+\beta)}\left\|e_{v}\right\|+\left\|p_{v}\right\|
$$

$$
\begin{aligned}
\|z(t)\| & \leq k_{1} e^{-k_{2}\left(t-t_{0}\right)}\left\|z\left(t_{0}\right)\right\|+k_{1} \int_{t_{0}}^{t} e^{-k_{2}\left(t-t_{0}\right)}\left((\sqrt{m}+1)\left\|p_{v}\right\|\right. \\
& \left.+\sqrt{m} N e^{-\rho(t-\tau)}\right) d \tau
\end{aligned}
$$

The result is available by calculation

$$
\|z(t)\| \leq k_{1} e^{-k_{2}\left(t-t_{0}\right)}\left\|z\left(t_{0}\right)\right\|+\frac{(\sqrt{m}+1) k_{1}}{k_{2}}\left\|p_{v}\right\|\left(1-e^{-k_{2}\left(t-t_{0}\right)}\right)+\frac{k_{1} \sqrt{m} N}{k_{2}-\rho}\left(e^{-\rho\left(t-t_{0}\right)}-e^{-k_{2}\left(t-t_{0}\right)}\right)
$$

Taking limits for $t \rightarrow \infty$, we get

$$
\|z(t)\| \rightarrow \Delta
$$

Namely, Multiple intelligences form target formations within the range of error $\Delta$

In the event driven control, in addition to the controller design, the most important thing is to exclude the Zeno phenomenon, that is, to determine the interval between the events of the two events, there is a positive lower bound. If the Zeno phenomenon cannot be ruled out, the advantages of event-driven control in saving resources and reducing computer burden can not be shown. That means that the designed event driven control failed.

Theorem 4.2. Under the control strategy (3.2), the trigger sequence is determined by the driving function (3.3), the closed-loop system does not exhibit Zenobehavior. Namely

$$
t_{k+1}^{i}-t_{k}^{i} \geq \ln \left(1+\frac{\theta}{\eta+r}\right)
$$

Proof. Because our aim is to exclude Zeno-behavior, $f_{i}\left(t, e_{i x}(\mathrm{t}), e_{i x}(\mathrm{t})\right) \leq 0, \mathrm{t} \in\left[t_{k}^{i}, t_{k+1}^{i}\right)$

$$
\begin{aligned}
& \sqrt{\alpha(\alpha+\beta)}\left|e_{\mathrm{ix}}\right|+\sqrt{\beta(\alpha+\beta)}\left|e_{i v}\right| \unlhd P_{i v} \mid+e^{-\rho\left(t-t_{0}\right)} \\
& \left|e_{\mathrm{ix}}\right|+\left|e_{\mathrm{iv}}\right| \leq \frac{1}{\sqrt{g(\alpha+\beta)}}\left(\left|P_{i v}\right|+e^{-\rho\left(t-t_{0}\right)}\right)
\end{aligned}
$$

where $g=\{\alpha, \beta\}_{\min }$ and a sufficient condition is given 


$$
\begin{gathered}
\text { Yu-lai Zheng } \\
\left|\dot{e}_{\mathrm{ix}}(t)\right| \leq\left|e_{\mathrm{iv}}(t)\right|+\left|v_{i}\left(t_{k}^{i}\right)\right| \\
\left|\dot{e}_{\mathrm{ix}}(t)\right| \leq\left|e_{\mathrm{iv}}(t)\right|+\frac{1}{\sqrt{\mathrm{g}(\alpha+\beta)}}\left(\left|p_{i v}\right|+1\right)+\left|v_{i}\left(t_{k}^{i}\right)\right|
\end{gathered}
$$

By solving the above differential equation, we can get

$$
\left|e_{\mathrm{ix}}(t)\right| \leq\left(\frac{1}{\sqrt{\mathrm{g}(\alpha+\beta)}}\left(\left|p_{i v}\right|+1\right)+\left|v_{i}\left(t_{k}^{i}\right)\right|\right)\left(e^{\left(t-t_{k}^{i}\right)}-1\right)
$$

Similarly, we can obtain:

$$
\begin{gathered}
\left|\dot{e}_{\mathrm{iv}}(t)\right| e_{i v}(t)+\sum_{j \in N_{i}} \alpha x_{i}\left(t_{k}^{i}\right)-p_{i d}-\left(x_{j}\left(t_{k}^{j}\right)-p_{j d}\right)+\beta x_{i}\left(t_{k}^{i}\right)-p_{i d}-\left(x_{j}\left(t_{k}^{j}\right)-p_{j d}\right) \\
\left|e_{\mathrm{iv}}(t)\right| \leq m\left(e_{i v}^{\left(t-t_{k}^{i}\right)}-1\right)
\end{gathered}
$$

where

$$
m=\sum_{j \in N_{i}} c\left|x_{i}\left(t_{k}^{i}\right)-p_{i d}-\left(x_{j}\left(t_{k}^{j}\right)-p_{j d}\right)\right|+\beta\left|x_{i}\left(t_{k}^{i}\right)-x_{j}\left(t_{k}^{j}\right)\right|
$$

$t_{k^{\prime}}^{j}$ represents the latest update time of neighbor $j$.

$$
\begin{aligned}
\eta_{k}^{i} & \triangleq \frac{1}{\sqrt{g(\alpha+\beta)}}\left(\left|p_{i v}\right|+e^{-\rho\left(t-t_{0}\right)}\right) \\
& =\left|e_{\mathrm{ix}}(t)\right|+\left|e_{\mathrm{iv}}(t)\right| .
\end{aligned}
$$

Because $\mathrm{e}^{-\rho\left(t-t_{0}\right)}$ is greater than zero, we can get

$$
\eta_{k}^{i} \geq \min \frac{\left|p_{i v}\right|}{\sqrt{y(\alpha+\beta)}} \triangleq \theta
$$

Similarly, we have

$$
\eta_{k}^{i} \leq(\eta+r)\left(e^{\left(t_{k+1}^{i}-t_{k}^{i}\right)}-1\right)
$$

In summary, we get

$$
t_{k+1}^{i}-t_{k}^{i} \geq \ln \left(1+\frac{\theta}{\eta+r}\right)
$$

Hence, Zeno-behavior can be excluded. Then, we have $t_{k+1}^{i}-t_{k}^{i} \geq \ln \left(1+\frac{\theta}{\eta+r}\right)$.

\section{Numerical results}

In this section, a numerical example is given to illustrate the feasibility and effectiveness of our theoretical results. We assume that there are ten agents in the multiagent system. Give the target position of 10 agents;

$$
\begin{gathered}
p_{i}(t)=r *[\sin (w * t+2 * p i / 5), w * \cos (w * t+2 * p i / 5), \\
\cos (w * t+2 * p i / 5),-w * \sin (w * t+2 * p i / 5)]^{\prime}
\end{gathered}
$$

where $r=5, w=0.5$ represent radius and speed respectively. 
A Fixed-Formation Control Based on Event-Driven of Multi-Agent System

$$
\mathrm{L}=\left[\begin{array}{cccccccccc}
2 & -1 & 0 & 0 & 0 & 0 & 0 & 0 & 0 & -1 \\
-1 & 2 & -1 & 0 & 0 & 0 & 0 & 0 & 0 & 0 \\
0 & -1 & 2 & -1 & 0 & 0 & 0 & 0 & 0 & 0 \\
0 & 0 & -1 & 2 & -1 & 0 & 0 & 0 & 0 & 0 \\
0 & 0 & 0 & -1 & 2 & -1 & 0 & 0 & 0 & 0 \\
0 & 0 & 0 & 0 & -1 & 2 & -1 & 0 & 0 & 0 \\
0 & 0 & 0 & 0 & 0 & -1 & 2 & -1 & 0 & 0 \\
0 & 0 & 0 & 0 & 0 & 0 & -1 & 2 & -1 & 0 \\
0 & 0 & 0 & 0 & 0 & 0 & 0 & -1 & 2 & -1 \\
0 & 0 & 0 & 0 & 0 & 0 & 0 & 0 & -1 & 2
\end{array}\right]
$$

Within the bounds of Theorem 4.1, take $\alpha=0.8, \beta=0.5$ multiple agents can achieve consistency within a certain range of errors. Figure 1 shows the trajectories of a multi-agent in a given time, figures two and three represent the control inputs of a multi-agent, Figure four shows the relation between the error and the threshold in the driving function.

\section{Conclusion}

In this paper, the two order formation problem of multi-agent system and the event driven control mechanism are discussed. We provide the effectiveness of the proposed control strategy by theoretical deduction and simulation examples. We can see from the simulation of Fig. 4 event-based control has great influence on updates of number and energy, therefore, how to design the driving conditions or changing driving conditions is a direction worthy of study. In addition, this paper finally achieves the consistency within a certain range of error, therefore the next step is to change the driving conditions so that they can achieve complete consensus.

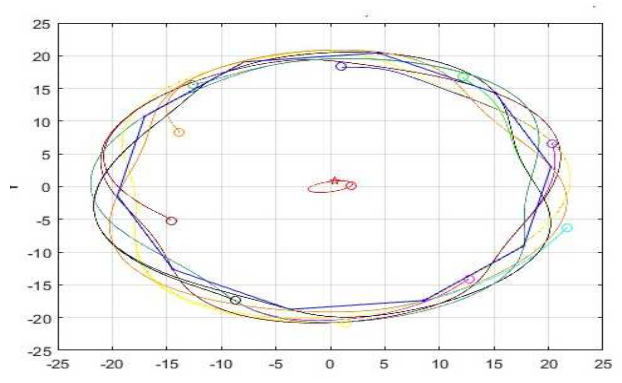

Figure 1: Move trajectory

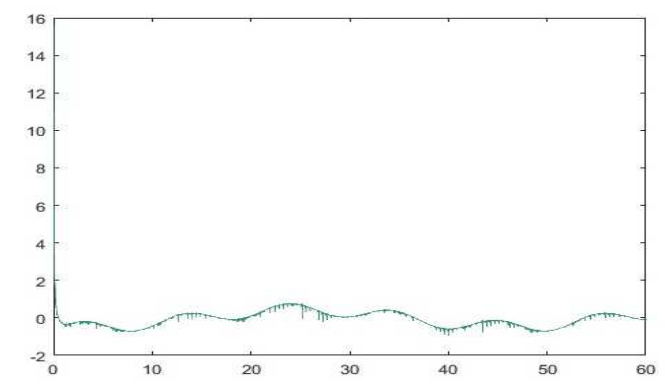

Figure 2: Distributed control 
Yu-lai Zheng

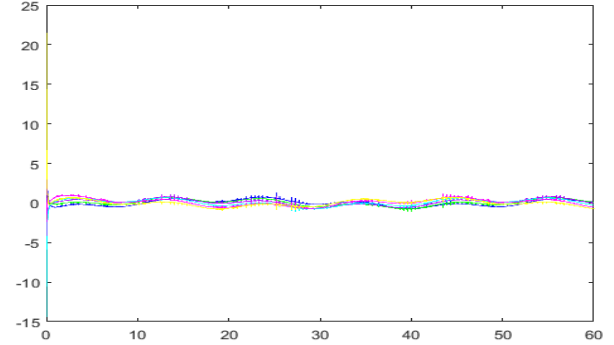

Figure 3: Velocity states

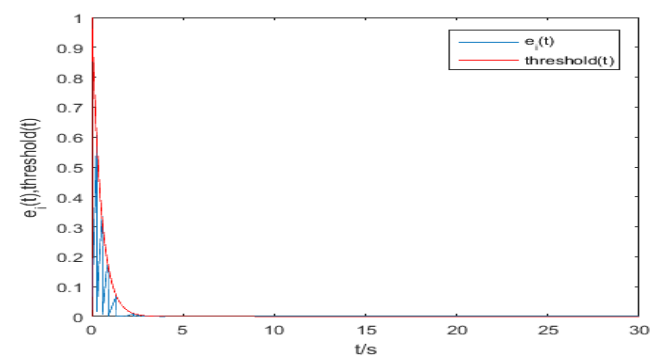

Figure 4: Error and threshold

\section{REFERENCES}

1. X.Wang, V.Yadav and S.N.Balakrishnan, Cooperative UAV formation flying with obstacle collision avoidance, IEEE Transactions on Control Systems Technology, 15 (2007) 672-679.

2. A.Dhariwal, G.Sukhatme and A.A.G.Requicha, Bacterium-inspired robots for environmental monitoring, IEEE International Conference on Robotics and Automation, 2 (2004) 1436 - 1443.

3. N.Nigam, S.Bieniawski, I.Kroo et al., Control of multiple UAVs for persistent surveillance: Algorithm and flight test results, IEEE Transactions on Control Systems Technology, 20 (2012) 1236-1251.

4. T.Kopfstedt, M.Mukai, M.Fujita et al., Control of formations of UAVs for surveillance and reconnaissance missions, IFAC Proceedings, Vol. 41 (2008) 51615166.

5. B.M.Chen, Y.L.Kai and H.L.Tong, Design and implementation of a leader-follower cooperative control system for unmanned helicopters, Control Theory and Technology, 8 (2010) 61-68.

6. K.Seungkeun and K.Youdan, Optimum design of three-dimensional behavioural decentra-Lized controller for UAV formation flight, Engineering Optimization, 41 (2009) 199-224.

7. M.Bayezit and B.Fidan, Distributed cohesive motion control of flight vehicle formations, IEEE Transactions on Industrial Electronics, 60 (2013) 5763-5772.

8. W.Zhu, H.Li and Z.Jiang, Consensus of multi-agent systems with time-varying topology: An event-based dynamic feedback scheme, International Journal of Robust and Nonlinear Control, 2016.

9. Y.Cao, W.Yu, W.Ren et al., An overview of recent progress in the study of distributed multi-agent coordination, IEEE Transactions on Industrial Informatics, 9 (2012) 427-438.

10. C.Wang, G.Xie, M.Cao et al., Circle formation for anonymous mobile robots with order preservation, 22 (2012) 1433-1438.

11. W.Zhu, Z.P.Jiang and G.Feng, Event-based consensus of multi-agent systems with general linear models, Automatica, 50 (2014) 552-558.

12. Y.Pan, S.Cao and L.Li, Constaints on interacting dark energy from time delay lenses, International Journal of Modern Physics D, 25 (2016) 1-8.

13. C.Wang, G.Xie, M.Cao et al., Circle formation for anonymous mobile robots with 
A Fixed-Formation Control Based on Event-Driven of Multi-Agent System order preservation, 22 (2012) 1433-1438.

14. H.Chen, K.Chang and C.S.Agate, UAV path planning with tangent-plus-lyapunov vector field guidance and obstacle avoidance, IEEE Transactions on Aerospace Electronic Systems, 49 (2013) 840-856.

15. W.Zhu and Z.P.Jiang, Event-based leader-following consensus of multi-agent systems with input time delay, IEEE Transactions on Automatic Control, 60 (2015) 1362-1367.

16. H.M.Guzey, N.Vignesh and S.Jagannathan et al., Distributed consensus-based eventtriggered approximate control of nonholonomic mobile robot formations, American Control Conference, 3 (2017) 3194-3199. 